\title{
Exploiting the hierarchical morphology of single-walled and multi-walled carbon nanotube films for highly hydrophobic coatings
}

\author{
Francesco De Nicola ${ }^{* 1,2}$, Paola Castrucci ${ }^{1,2}$, Manuela Scarselli ${ }^{1,2}$, Francesca Nanni ${ }^{3}$, \\ Ilaria Cacciotti ${ }^{4}$ and Maurizio De Crescenzi ${ }^{1,2,5}$
}

\section{Full Research Paper}

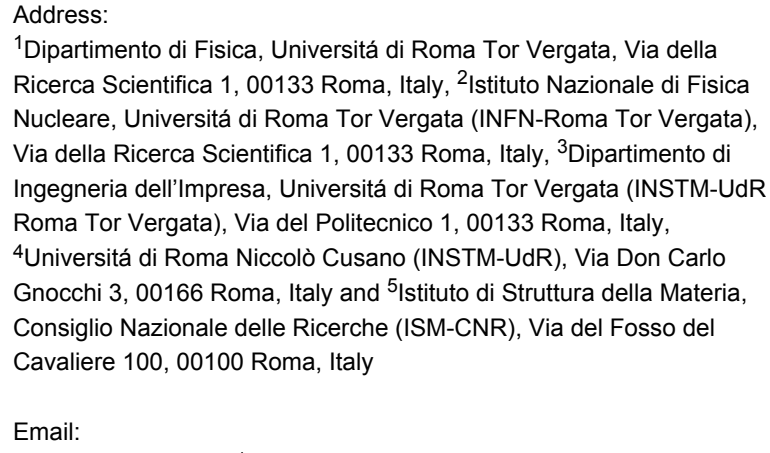

${ }^{1}$ Dipartimento di Fisica, Universitá di Roma Tor Vergata, Via della Ricerca Scientifica 1, 00133 Roma, Italy, ${ }^{2}$ Istituto Nazionale di Fisica Nucleare, Universitá di Roma Tor Vergata (INFN-Roma Tor Vergata), Via della Ricerca Scientifica 1, 00133 Roma, Italy, ${ }^{3}$ Dipartimento di Ingegneria dell'Impresa, Universitá di Roma Tor Vergata (INSTM-UdR Roma Tor Vergata), Via del Politecnico 1, 00133 Roma, Italy, ${ }^{4}$ Universitá di Roma Niccolò Cusano (INSTM-UdR), Via Don Carlo Gnocchi 3, 00166 Roma, Italy and ${ }^{5}$ Istituto di Struttura della Materia, Consiglio Nazionale delle Ricerche (ISM-CNR), Via del Fosso del Cavaliere 100, 00100 Roma, Italy

Email:

Francesco De Nicola* - fdenicola@roma2.infn.it

* Corresponding author

\section{Keywords:}

hierarchical structures; hydrophobic surfaces; multi-walled carbon nanotube; single-walled carbon nanotube; wetting transitions

\author{
Beilstein J. Nanotechnol. 2015, 6, 353-360. \\ doi:10.3762/bjnano.6.34 \\ Received: 12 September 2014 \\ Accepted: 13 January 2015 \\ Published: 02 February 2015 \\ This article is part of the Thematic Series "Self-assembly of \\ nanostructures and nanomaterials". \\ Guest Editor: I. Berbezier \\ (c) 2015 De Nicola et al; licensee Beilstein-Institut. \\ License and terms: see end of document.
}

\begin{abstract}
Self-assembled hierarchical solid surfaces are very interesting for wetting phenomena, as observed in a variety of natural and artificial surfaces. Here, we report single-walled (SWCNT) and multi-walled carbon nanotube (MWCNT) thin films realized by a simple, rapid, reproducible, and inexpensive filtration process from an aqueous dispersion, that was deposited at room temperature by a dry-transfer printing method on glass. Furthermore, the investigation of carbon nanotube films through scanning electron microscopy (SEM) reveals the multi-scale hierarchical morphology of the self-assembled carbon nanotube random networks. Moreover, contact angle measurements show that hierarchical SWCNT/MWCNT composite surfaces exhibit a higher hydrophobicity (contact angles of up to $\left.137^{\circ}\right)$ than bare SWCNT $\left(110^{\circ}\right)$ and MWCNT $\left(97^{\circ}\right)$ coatings, thereby confirming the enhancement produced by the surface hierarchical morphology.
\end{abstract}




\section{Introduction}

In general, the surface morphology [1] is a crucial parameter for the fabrication of artificial hydrophobic surfaces and may be enhanced especially by hierarchical [2-7] and fractal structures $[7,8]$, possibly allowing for the formation of air pockets to further impede the penetration of water [9].

In particular, hierarchical surface morphologies are a recent concept introduced to explain the wetting properties of surfaces such as plant leaves [2,3], bird feathers [10], and insect legs [11]. These surfaces are made of a hierarchical micro- and nanomorphology which improves their wettability.

It is indeed well-established $[12,13]$ that on composite rough surfaces a hierarchical morphology may induce a wetting transition from Wenzel [1] to Cassie-Baxter [9] state owing to air trapping. Moreover, this transition may occur by passing through thermodynamically metastable states [13-16], where the free energy surface presents one absolute minimum and one or more local minima separated from the former by large free energy barriers, as compared to the thermal energy. Metastability can also have a technological importance, as in practice, it represents a way of extending the range of stability of the Cassie-Baxter state $[14,17]$. Conversely, a negative consequence of metastability is that it might prevent or slow down the transition between Wenzel and Cassie-Baxter states [14,17].

Moreover, biomimetics [18,19] may be exploited in order to realize cutting edge artificial surfaces $[2,3,5]$ that mimicking natural surface. In this way these surfaces can be optimized for hydrophobic (lipophilic) and/or hydrophilic (lipophobic) applications.

Motivated by this concept, we report here the fabrication of highly hydrophobic coatings of self-assembling SWCNTs on MWCNTs. Since the former are smaller than the latter (about one order of magnitude), we observed that a surface hierarchy naturally occurs by depositing layer by layer a SWCNT film upon a MWCNT film. The particular two-fold hierarchical morphology of the surface, resembling that observed in lotus leaves [3] and rose petals [2] in which micropapillae are made of nanopapillae, improves the hydrophobic behavior of carbon nanotube coatings compared to bare SWCNT and MWCNT films. Moreover, we report for the first time the experimental Wenzel/Cassie-Baxter phase diagram $[8,12,17]$ for a carbon nanotube surface, showing that the transition between the Wenzel and Cassie-Baxter states occurs by passing through metastable states.

Generally, carbon nanotubes $[20,21]$ are the one-dimensional allotropic form of carbon with cylindrical symmetry and a sp ${ }^{2}$ lattice. Carbon nanotubes may be single-walled or multi-walled depending on the number of coaxially arranged graphite planes. Moreover, owing to their honeycomb lattice, carbon nanotubes are inherently hydrophilic (the contact angle of graphite with water being approx. $\left.86^{\circ}[22]\right)$ but apolar. However, by surface functionalization or textured arrangement it can be possible to realize carbon nanotube films which offer versatility, high stability, and multi-functionality owing to their exceptionally unique properties [21], making their usage widespread in hydrophobic surface applications [4,5,23-35].

Furthermore, self-assembly hierarchical nanostructured materials [36-39] are nowadays investigated as a consequence of their tunable peculiar properties and the easy, highly reproducible, and low-cost fabrication. In addition, they are ideal low-dimensional materials for the fabrication of high aspect ratio and large area devices [40].

\section{Results and Discussion}

The films obtained from the process described in the Experimental section are porous random networks of SWCNTs and MWCNTs that exhibit a hierarchical morphology made of micro- and nanostructures, as evident from SEM micrographs in Figure 1. From SEM image analysis (see Experimental section), we estimated the pore radius $\rho$ and the bundle diameter $d$ of the SWCNT and MWCNT random networks. The obtained results are reported in Table 1 together with the SWCNT microstructure area $S$ and height $h$. However, in the case of MWCNT films, no microstructures were observed. It is noteworthy that the characteristic dimension $d$ of MWCNTs is bigger by about one order than that of SWCNTs.

In particular, we considered the microstructures shown in Figure 1c as ripples randomly distributed within the film. Such self-assembly occurs by an out-of-plane bending process during evaporative drying of single-walled carbon nanotube film during its preparation $[36,37,41]$. The out-of-plane assembly is the result of the competition between attractive capillary forces and bending stress due to the elasticity of SWCNT film. Once the liquid is completely evaporated, a pattern of micrometersized randomly shaped islands is formed. If, after complete evaporation, there is a balance between adhesion and elastic energy, the microstructures are in a stable bent configuration with respect to further wetting-dewetting cycles. This selfassembly leads to an intrinsic hierarchical microstructured (ripples) and nanostructured (carbon nanotubes) roughness able to enhance the wetting properties of the SWCNT film. Conversely, the MWCNT sample (Figure 1d) just aligned vertically out of plane. 
(a)

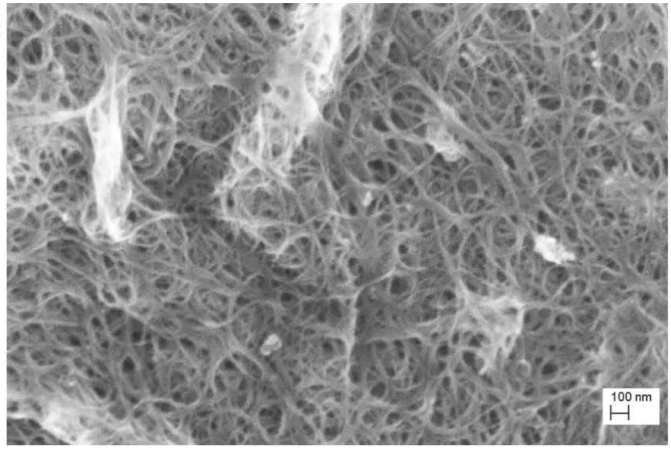

(c)

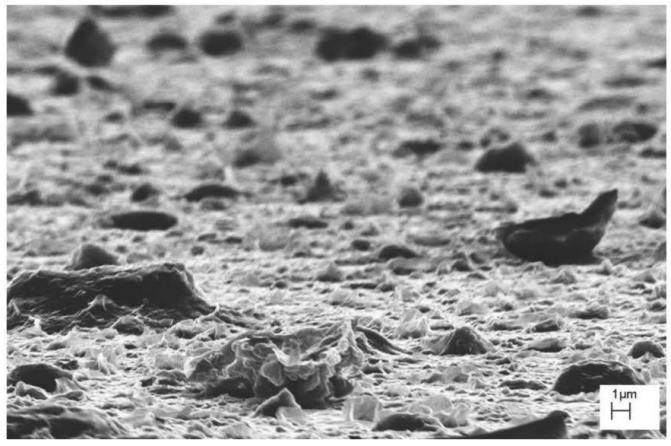

(b)

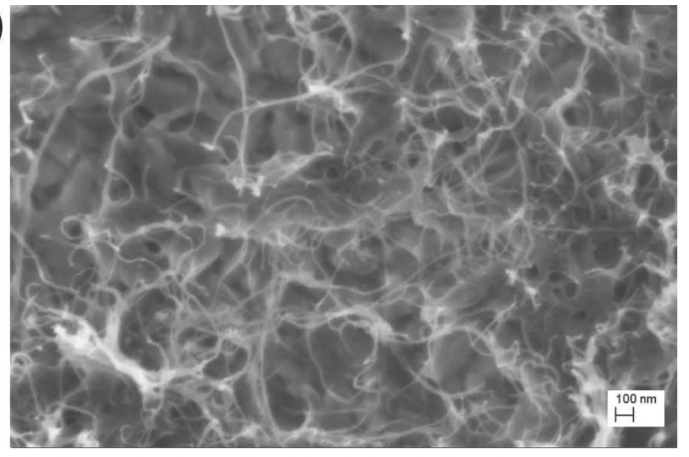

(d)

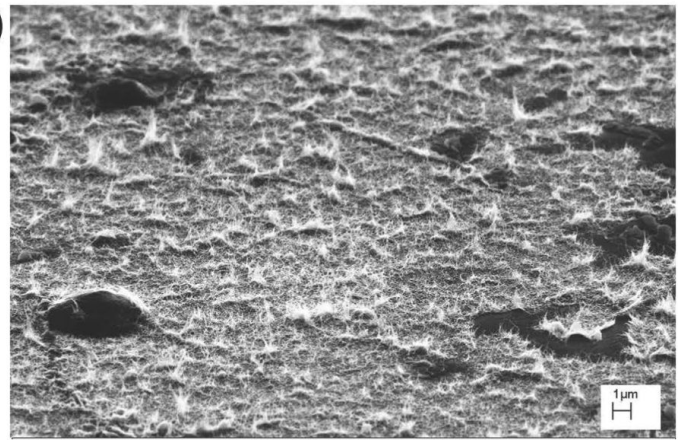

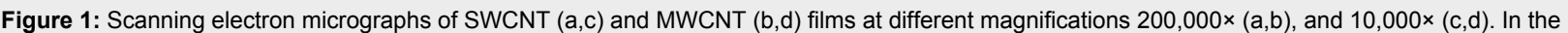
images taken at grazing incidence (c,d) it is possible to observe that SWCNTs (c) self-assemble in ripples forming several microstructures, while MWCNTs (d) just aligned in the out-of-plane vertical direction. (d) Black areas are holes in the film.

\begin{tabular}{|c|c|c|c|c|c|}
\hline sample & $\rho(\mathrm{nm})$ & $d(\mathrm{~nm})$ & $S\left(\mu m^{2}\right)$ & $h(\mu \mathrm{m})$ & $\theta\left({ }^{\circ}\right)$ \\
\hline SWCNT & $2-8$ & $4-8$ & $0.003-0.007$ & $1.6-11.7$ & $110 \pm 3$ \\
\hline MWCNT & $40-44$ & $34-84$ & - & - & $97 \pm 8$ \\
\hline SWCNT/MWCNT & $47-51$ & - & $7.3-13.7$ & $3.2-61.6$ & $129 \pm 8$ \\
\hline MWCNT/SWCNT & $5-7$ & - & $7.7-14.3$ & $2.3-76.2$ & $103 \pm 7$ \\
\hline
\end{tabular}

Furthermore, we induced an extrinsic hierarchical architecture by depositing a SWCNT film on a MWCNT film (SWCNT/ MWCNT) and in reverse order (MWCNT/SWCNT), as shown in Figure 2. From SEM image analysis, we obtained the two film pore diameters, microstructure areas and heights, as reported in Table 1. In both cases, a self-assembly led to the formation of several huge microstructures as compared to the those of the SWCNT films.

Moreover, in Figure 3a and Figure 3b, images of water droplets cast on our SWCNT and MWCNT films are shown, with average contact angle values of $\theta=110 \pm 3^{\circ}$ and $\theta=97 \pm 8^{\circ}$, respectively. These results can be ascribed to the particular morphology of both the films induced by the inherent properties of the carbon nanotubes (e.g., self-assembly, nanotube diameter and spatial orientation) and film preparation method. We also found that for the SWCNT/MWCNT sample the extrinsic surface hierarchy increased the hydrophobicity of the MWCNT sample, exhibiting a highly hydrophobic average contact angle value of $\theta=129 \pm 8^{\circ}$ (Figure 3c), comparable to the values of PTFE (Teflon) of $108-118^{\circ}[22,42]$. Conversely, for the MWCNT/SWCNT sample (Figure 3d) a slightly decrease of the average contact angle value $\left(\theta=103 \pm 7^{\circ}\right)$ with respect to the bare SWCNT sample was encountered.

Our results, summarized in Table 1, may be interpreted on the basis of the microstructure characteristic dimensions $S$ and $h$. In both the SWCNT/MWCNT and MWCNT/SWCNT samples the microstructure characteristic dimensions are comparable with those of lotus and rose micropapillae [2,3]. Nevertheless, in the latter the extrinsic hierarchical morphology is reversed (bigger MWCNT scale superimposed on the smaller SWCNT scale), thereby losing the hierarchical fakir effect $[7,43]$. Therefore, the SWCNT/MWCNT sample has the best hydrophobic behavior 
(a)

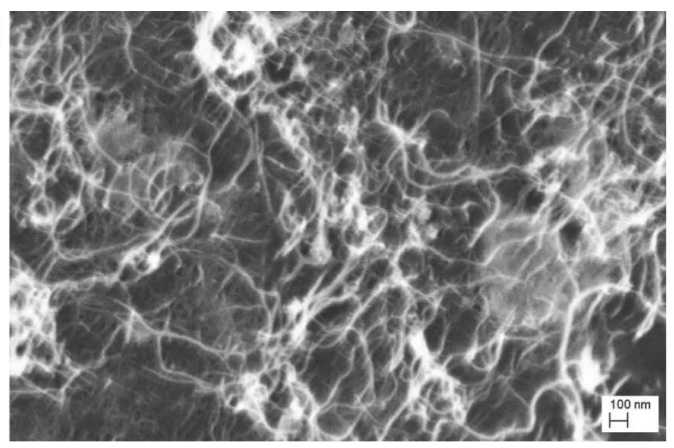

(c)

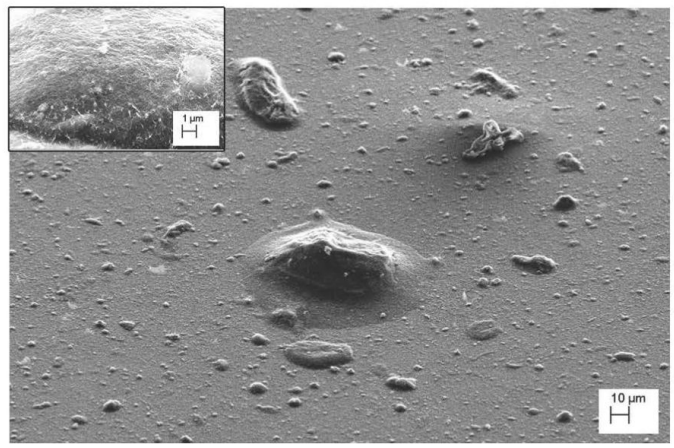

(b)

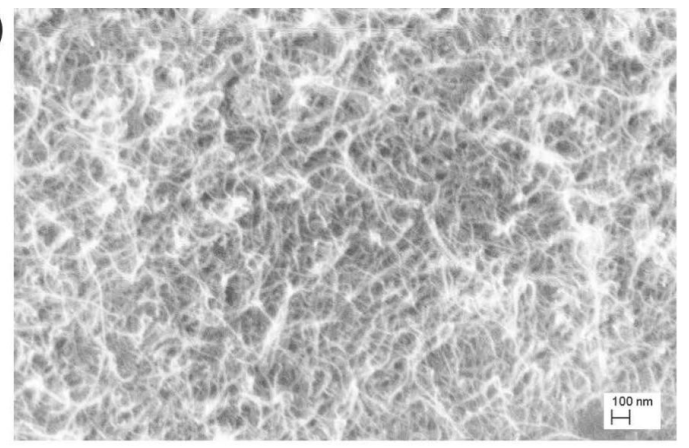

(d)

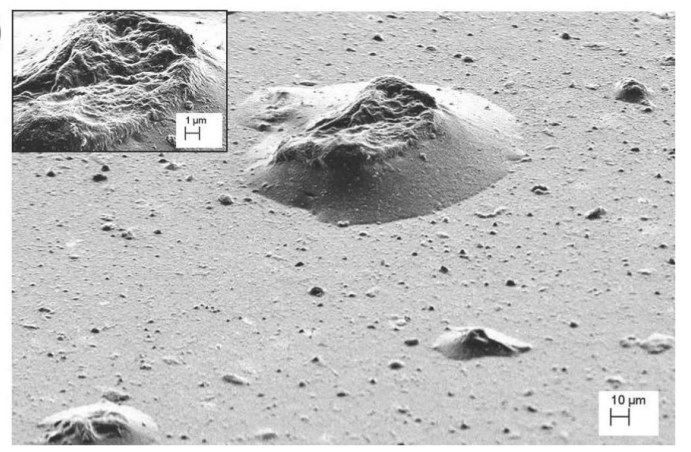

Figure 2: Scanning electron micrographs of SWCNT/MWCNT $(a, c)$ and MWCNT/SWCNT $(b, d)$ films at different magnifications $200,000 \times(a, b)$, $1,000 \times(c, d)$, and 10,000× (c,d insets). In the images taken at grazing incidence (c,d and insets), it is possible to observe that in both cases the selfassembly forms several huge microstructures. (c,d insets) Details of the microstructures showing a hierarchical morphology very similar to that of lotus leaves and rose petals.
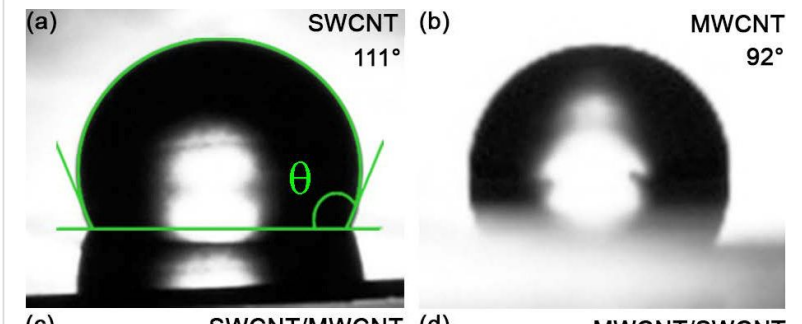

(c)

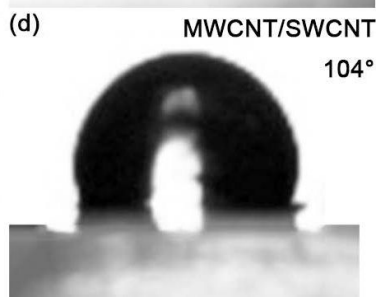

Figure 3: Water droplets cast on SWCNT (a), MWCNT (b), SWCNT/ MWCNT (c), and MWCNT/SWCNT (d) films. Owing to the rough and porous surface of the samples, water drops exhibit different contact angle values, depending on the exact place at the surface on which they are cast. In this case, the contact angle can be only defined on average.

because is the most biomimetic. We remark that the large deviation of the average value of the contact angle is due to the highly rough and porous surface of our samples. In addition, no roll-off angle value could be measured, evidently due to the high contact angle hysteresis, which pinned the droplets to the surface [2].

In order to better understand the origin of the enhancement provided by the SWCNT/MWCNT film over the MWCNT film, we characterized the wetting state of the former composite surface with respect to the latter. In Figure $4 a$, we report the contact angle of both films as a function of the concentration in volume percent of ethanol in water. It is possible to observe that since ethanol has a lower liquid-vapor surface tension $\left(\gamma_{\mathrm{LV}}=22 \mathrm{~mJ} \cdot \mathrm{m}^{-2}\right)$ than water $\left(\gamma_{\mathrm{LV}}=72 \mathrm{~mJ} \cdot \mathrm{m}^{-2}\right)$, the higher the ethanol concentration in water, the lower the surface tension of the solution. Furthermore, the contact angle is generally proportional to the liquid surface tension by the Young's relation

$$
\cos \theta=\frac{\gamma_{S V}-\gamma_{S L}}{\gamma_{L V}},
$$

where $\gamma_{\mathrm{SV}}$ and $\gamma_{\mathrm{SL}}$ are the solid-vapor and solid-liquid surface tensions, respectively. Therefore, also the contact angles of the carbon nanotube films decrease with the decrease in surface tension of the liquid droplet. This phenomenon is connected to the lipophilicity of the apolar surface of the carbon nanotube. Indeed, on our carbon nanotube films no contact angle $(\theta \approx 0)$ can be measured for pure ethanol droplets. Therefore, we 

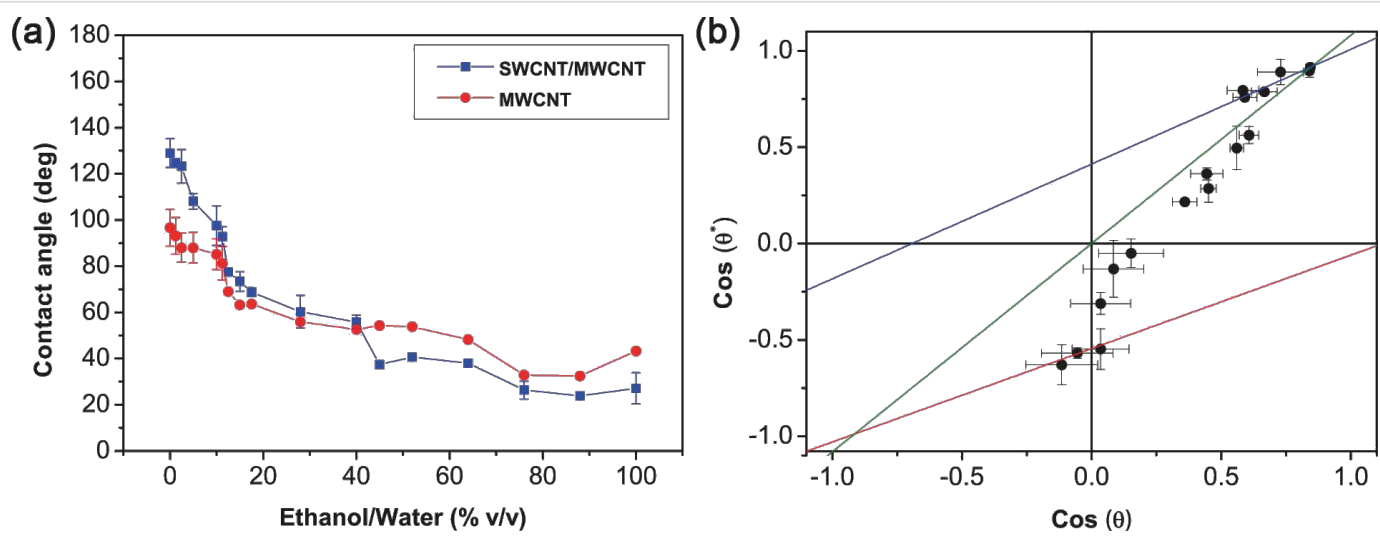

Figure 4: (a) Contact angle of the SWCNT/MWCNT (blue squares) and MWCNT (red dots) films as a function of ethanol concentration in water. (b) Wenzel/Cassie-Baxter phase diagram of the SWCNT/MWCNT surface respect to the MWCNT surface. Wetting states are studied changing the liquid surface tension by adding different ethanol concentrations in water. Wenzel regime (green solid line) fit reports a roughness factor $r=1.08 \pm 0.01$, while lipophilic (blue solid line) and hydrophobic (red solid line) Cassie-Baxter regime fits report respectively a liquid fraction $\phi_{+}=0.41 \pm 0.04$ and an air fraction $\phi_{-}=0.54 \pm 0.02$. The Wenzel/Cassie-Baxter transition point in the hydrophobic regime is the intersection between the red and green solid lines, while in the lipophilic regime it is the intersection between the blue and green solid lines. Error bars are standard deviations.

investigated all the wetting phenomena occurring on our carbon nanotube surface, exploring all the wetting states. We further noted that for $\theta \approx 56^{\circ},(\cos \theta \approx 0.56)$ there is an intersection point between the two curves in Figure $4 \mathrm{a}$, beyond which the SWCNT/MWCNT surface becomes more lipophilic than the MWCNT surface. That point corresponds to the transition point from Wenzel to Cassie-Baxter state in the lipophilic region of the Wenzel/Cassie-Baxter phase diagram, as confirmed from the plot (first quadrant) in Figure 4b. However, the plot in Figure $4 \mathrm{~b}$ shows that the transition occurs by passing through metastable states with an abrupt change in the wetting state. We fitted our data with the lipophilic Cassie-Baxter's equation [9]

$$
\cos \theta^{*}=\left(1-\phi_{+}\right) \cos \theta+\phi_{+}, \quad 1=\phi+\phi_{+},
$$

with $\phi$ the surface solid fraction, $\phi_{+}$the surface fraction wetted by the liquid, $\theta^{*}$ the SWCNT/MWCNT surface contact angle and $\theta$ the MWCNT surface contact angle. We obtained from fit a liquid fraction $\phi_{+}=0.41 \pm 0.04$ in contact with the droplet. However, we remark that these metastable Cassie-Baxter states coexist with the Wenzel states, which are stable because lower in surface free energy.

Moreover we fitted our data in Figure $4 \mathrm{~b}$ with Wenzel's equation [1]

$$
\cos \theta^{*}=r \cos \theta, \quad r \geq 1,
$$

where $r$ is the roughness factor (i.e, the ratio between the actual wet surface area and its projection on the plane). Interestingly, the fit returned $r=1.08 \pm 0.01$, which means that substantially the SWCNT/MWCNT sample has the same roughness of the MWCNT sample. It is noteworthy that in our case $r \approx 1$ does not mean that the surface is smooth, because we are not comparing the SWCNT/MWCNT with its corresponding smooth surface with the same chemistry, such as plain graphite. However, in the latter case we would have had a high roughness factor [41]. Therefore, we can exclude a roughness enhancement, which we did not observe, as the reason of a such improvement in hydrophobic behavior the SWCNT/MWCNT sample over the MWCNT sample. In addition, from the relation [44]

$$
\cos \theta^{\prime}=\frac{1-\phi_{+}}{r-\phi_{+}}
$$

we can infer that the lipophilic Wenzel/Cassie-Baxter transition point is $\cos \theta^{\prime}=0.88$ (the intersection between the blue and green solid lines in Figure $4 \mathrm{~b}$ ), which is beyond the measured data, thus confirming that the achieved lipophilic Cassie-Baxter states are metastable.

Conversely, in the hydrophobic region (third quadrant of the plot) we observe a sharp discontinuity beyond $\cos \theta=0$, confirming again that the transition between the Wenzel and Cassie-Baxter states is not continuous, but it undergoes metastable states which slow down the dewetting process. Actually, by fitting our data in Figure $4 \mathrm{~b}$ with the hydrophobic Cassie-Baxter's equation

$$
\cos \theta^{*}=\left(1-\phi_{-}\right) \cos \theta-\phi_{-}, \quad 1=\phi+\phi_{-},
$$


we obtained an air surface fraction $\phi_{-}=0.54 \pm 0.02$ below the liquid droplet. Furthermore, by the relation [44]

$$
\cos \theta^{\prime \prime}=\frac{\phi_{-}-1}{r-\phi_{-}},
$$

we can infer that the hydrophobic Wenzel/Cassie-Baxter transition point is $\cos \theta^{\prime \prime}=-0.85$ (the intersection between the red and green solid lines in Figure $4 \mathrm{~b}$ ), which is beyond the measured data, thus confirming that the achieved hydrophobic Cassie-Baxter states are metastable. Nevertheless, this result suggests a consistent air pocket formation [9].

Therefore, we can assert that the only cause of the improved hydrophobicity/lipophilicity of the SWCNT/MWCNT film over the MWCNT film, is the fakir effect induced by the two-fold hierarchical morphology given by the SWCNT film superimposed on the MWCNT film. This particular morphology induces the formation of air pocket when the interaction with the liquid is hydrophobic, otherwise it favourites the formation of a precursor liquid film [44] that enhances the wettability of the carbon nanotube surface, when the interaction with the liquid is lipophilic.

Furthermore, we studied the stability of our carbon nanotube films over time by performing suction experiments. Figure 5 reports the variations of the contact angle value as a function of the elapsed time from dropping the liquid on the SWCNT, MWCNT, SWCNT/MWCNT, and MWCNT/SWCNT coatings. In such suction experiment, we show that although the samples are porous, the contact angle trend is quite constant. In particular, we demonstrated the stability over time of the hydrophobic Cassie-Baxter metastable state for the SWCNT/MWCNT sample. However, the slight linear decrease of the contact angle in time is both due to liquid evaporation and suction by the porous films. Our results are particularly remarkable, since the water contact angle of carbon nanotube films has been reported [45] so far to linearly decrease with time, from an initial value of approx. $146^{\circ}$ to about zero within $15 \mathrm{~min}$.

\section{Conclusion}

Single-walled and multi-walled carbon nanotube films were prepared by vacuum filtration of an aqueous dispersion. Such coatings were deposited by dry-transfer printing on glass, at room temperature. Furthermore, SEM images revealed the intrinsic hierarchical nature of carbon nanotube random networks owed to a dry-induced out-of-plane self-assembly phenomenon. Moreover, static contact angles of sessile water drops cast on carbon nanotube composite surfaces were measured, finding that our SWCNT random network films are more hydrophobic than our MWCNT random network films.

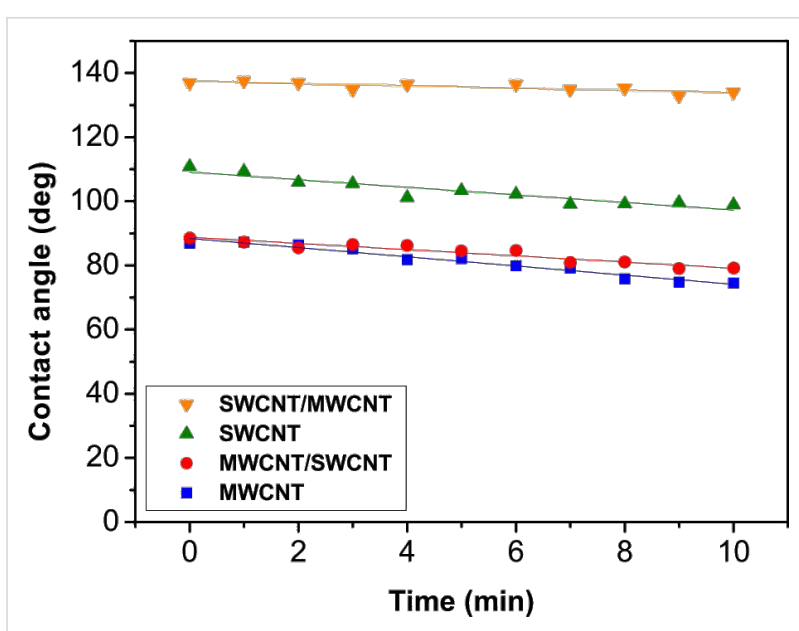

Figure 5: Variations of the contact angle as a function of the elapsed time from drop cast on the porous SWCNT (green triangles), MWCNT (blue squares), SWCNT/MWCNT (orange inverted triangles), MWCNT/ SWCNT (red dots) films. The quite constant trend of the SWCNT/ MWCNT contact angle value shows the stability in time of the carbon nanotube hydrophobic Cassie-Baxter metastable state.

This behavior may be ascribed to remarkable differences in the two film morphology induced by our preparation method. However, since the characteristic dimension of SWCNT is one order of magnitude smaller than MWCNT, when a SWCNT film is placed on a MWCNT film an extrinsic hierarchical morphology occurs making the resulting composite surface highly hydrophobic $\left(\theta=129 \pm 8^{\circ}\right)$. We showed that our results are due to two main reasons: (i) The characteristic dimension of the self-assembly microstructures in the SWCNT/MWCNT samples are comparable with those of micropapillae in hydrophobic plant leaves. (ii) The hierarchical surface morphology lead to the formation of a consistent amount of air pockets, as a consequence of the transition from the hydrophobic Wenzel state to the hydrophobic Cassie-Baxter metastable state. In addition, we observed that the latter state is fairly stable in time. Such highly hydrophobic hierarchical carbon nanotube coatings may be very attracting for several industrial applications such as waterproof surfaces [23], antisticking [31], anti-contamination [4], self-cleaning [46], antifouling [47], anti-fogging [48], low-friction coatings [5], adsorption [30], lubrication [22], dispersion [44], and selfassembly [49].

\section{Experimental}

\section{Fabrication of carbon nanotube films}

Highly pure SWCNT powder (Sigma-Aldrich, assay $>90 \%$, diameter: $0.7-0.9 \mathrm{~nm}$ ) and MWCNT powder (Nanocyl, NC7000, assay $>90 \%$, diameter: $5-50 \mathrm{~nm})$ were dispersed in aqueous solution $\left(80 \mu \mathrm{g} \cdot \mathrm{mL}^{-1}\right)$ with $2 \% \mathrm{w} / \mathrm{v}$ sodium dodecyl sulfate (Sigma-Aldrich, assay $>98.5 \%$ ) anionic surfactant. In addition, to better disperse the suspension, the carbon nanotubes 
were tip-ultrasonicated (Branson S250A, $200 \mathrm{~W}, 20 \%$ power, $20 \mathrm{kHz}$ ) in an ice-bath for an hour and the unbundled supernatant was collected through a pipette. The result was a welldispersed suspension that is stable for several months. Carbon nanotube films were fabricated by a vacuum filtration process of $1 \mathrm{~mL}$ in volume of the dispersion cast on mixed cellulose ester filters (Pall GN6, 1 in diameter, $0.45 \mu \mathrm{m}$ pore diameter). In order to prepare hierarchical MWCNT/SWCNT films, after filtering $1 \mathrm{~mL}$ of the SWCNT dispersion, $1 \mathrm{~mL}$ of the MWCNT dispersion was filtered. This process was also carried out in reverse order to produce SWCNT/MWCNT films. In this way, a stack of two different film layers were obtained. Subsequently, rinsing in water and in a solution of ethanol, methanol and water (15:15:70) to remove as much surfactant as possible was performed. Samples were made uniformly depositing by the dry-transfer printing method carbon nanotube films on Carlo Erba soda-lime glass slides. More details about this novel deposition technique without chemical deposition processes have been reported elsewhere [41].

\section{Sample characterization}

Scanning electron microscopy micrographs were acquired with Zeiss Leo Supra 35 field emission scanning electron microscope (FEG-SEM) and analyzed in order to measure carbon nanotube bundle diameter, network pore, and microstructure feature (height and area) distributions. A statistical analysis of these quantities was performed and the values reported in Table 1 were estimated by taking the quantity distribution mode values and standard deviations. In particular, we performed microstructure area measurements through analyzing the SEM micrographs of the films at a magnification of $30,000 \times$ with a threshold algorithm and considering their irregular shape. The analysis of microstructure height was carried out on SEM images acquired at a magnification of $10,000 \times$ at grazing angle, i.e., by tilting the sample to an angle very close to $90^{\circ}$ with respect to the sample normal. In such a way, the height of film microstructures can be estimated by trigonometric measurements. The film pore area defined as the area of the irregular empty regions delimited by the intersection among carbon nanotube bundles was quantified by the statistical analysis with a threshold algorithm of film SEM images at the highest magnification $(200,000 \times)$, at which pores are clearly observable. The radius of the pore was calculated by considering the pore area as that of a circle.

\section{Contact angle measurements}

Images of sessile water drops cast on carbon nanotube films were acquired by a custom setup with a CCD camera. Static advanced contact angles were measured by increasing the volume of the drop by steps of $1 \mu \mathrm{L}$, and a plugin [50] for the open-source software ImageJ was exploited to estimate the contact angle values. This plugin exploits an algorithm based on a small-perturbation solution of the Young-Laplace equation [22]. Furthermore, the presented method is applied to a continuous image of the droplet by using cubic B-Spline interpolation of the drop contour to reach subpixel resolution. Every contact angle value reported is the average over five measures of images of droplets cast on five different points of the film (namely in the center, north, south, east, and west part). The deionized water $(18.2 \mathrm{M} \Omega \cdot \mathrm{cm})$ drop volume used to achieve the contact angles of samples was $V=10 \mu \mathrm{L}$. Moreover, every contact angle was measured $15 \mathrm{~s}$ after drop casting to ensure that the droplet reached its equilibrium position.

\section{Acknowledgements}

The authors thank R. De Angelis, F. De Matteis, and P. Prosposito (Universitá di Roma Tor Vergata, Roma, Italy) for their courtesy of contact angle instrumentation. This project was financial supported by the European Office of Aerospace Research and Development (EOARD) through the Air Force Office of Scientific Research Material Command, USAF, under Grant No. FA9550-14-1-0047.

\section{References}

1. Wenzel, R. N. Ind. Eng. Chem. 1936, 28, 988-994. doi:10.1021/ie50320a024

2. Feng, L.; Zhang, Y.; Xi, J.; Zhu, Y.; Wang, N.; Xia, F.; Jiang, L. Langmuir 2008, 24, 4114-4119. doi:10.1021/la703821h

3. Sun, M.; Luo, C.; Xu, L.; Ji, H.; Ouyang, Q.; Yu, D.; Chen, Y. Langmuir 2005, 21, 8978-8981. doi:10.1021/la050316q

4. Li, Y.; Huang, X. J.; Heo, S. H.; Li, C. C.; Choi, Y. K.; Cai, W. P.; Cho, S. O. Langmuir 2007, 23, 2169-2174. doi:10.1021/la0620758

5. Jung, Y. C.; Bhushan, B. ACS Nano 2009, 3, 4155-4163. doi:10.1021/nn901509r

6. Egatz-Gomez, A.; Majithia, R.; Levert, C.; Meissner, K. E. RSC Adv. 2012, 2, 11472-11480. doi:10.1039/c2ra22267a

7. Bittoun, E.; Marmur, A. Langmuir 2012, 28, 13933-13942. doi:10.1021/la3029512

8. Shibuichi, S.; Onda, T.; Satoh, N.; Tsujii, K. J. Phys. Chem. 1996, 100, 19512-19517. doi:10.1021/jp9616728

9. Cassie, A. B. D.; Baxter, S. Trans. Faraday Soc. 1944, 40, 546-551. doi:10.1039/tf9444000546

10. Bormashenko, E.; Bormashenko, Y.; Stein, T.; Whyman, G.; Bormashenko, E. J. Colloid Interface Sci. 2007, 311, 212-216. doi:10.1016/j.jcis.2007.02.049

11. Gao, X.; Jiang, L. Nature 2004, 432, 36. doi:10.1038/432036a

12. Lafuma, A.; Quéré, D. Nat. Mater. 2003, 2, 457-460. doi:10.1038/nmat924

13. Giacomello, A.; Chinappi, M.; Meloni, S.; Casciola, C. M. Phys. Rev. Lett. 2012, 109, 226102. doi:10.1103/PhysRevLett.109.226102

14. Giacomello, A.; Meloni, S.; Chinappi, M.; Casciola, C. M. Langmuir 2012, 28, 10764-10772. doi:10.1021/la3018453

15. Savoy, E. S.; Escobedo, F. A. Langmuir 2012, 28, 16080-16090. doi:10.1021/la303407r

16. Murakami, D.; Jinnai, H.; Takahara, A. Langmuir 2014, 30, 2061-2067. doi:10.1021/la4049067 
17. Bico, J.; Thiele, U.; Quéré, D. Colloids Surf., A 2002, 206, 41-46. doi:10.1016/S0927-7757(02)00061-4

18. Bhushan, B. Philos. Trans. R. Soc. London, Ser. A 2009, 367, 1445-1486. doi:10.1098/rsta.2009.0011

19. Bar-Cohen, Y. Biomimetics Biologically Inspired Technologies; CRC Press: Boca Raton, 2005.

20. lijima, S. Nature 1991, 354, 56-58. doi:10.1038/354056a0

21. Jorio, A.; Dresselhaus, G.; Dresselhaus, M. S. Carbon Nanotubes Advanced Topics in the Synthesis, Structure, Properties and Applications; Springer: New York, 2008.

22. Adamson, A. W.; Gast, A. P. Physical Chemistry of Surfaces; John Wiley \& Sons: New York, 1997.

23. Georgakilas, V.; Bourlinos, A. B.; Zboril, R.; Trapalis, C. Chem. Mater. 2008, 20, 2884-2886. doi:10.1021/cm7034079

24. Wang, N.; Xi, J.; Wang, S.; Liu, H.; Feng, L.; Jiang, L. J. Colloid Interface Sci. 2008, 320, 365-368. doi:10.1016/j.jcis.2008.01.005

25. Yang, J.; Zhang, Z.; Men, X.; Xu, X.; Zhu, X. J. Colloid Interface Sci. 2010, 346, 241-247. doi:10.1016/j.jcis.2010.02.040

26. Kakade, B. A.; Pillai, V. K. J. Phys. Chem. C 2008, 112, 3183-3186. doi:10.1021/jp711657f

27. Nasibulin, A. G.; Kaskela, A.; Mustonen, K.; Anisimov, A. S.; Ruiz, V.; Kivistö, S.; Rackauskas, S.; Timmermans, M. Y.; Pudas, M.; Aitchison, B.; Kauppinen, M.; Brown, D. P.; Okhotnikov, O. G.; Kauppinen, E. I. ACS Nano 2011, 5, 3214-3221. doi:10.1021/nn200338r

28. Wang, C.-F.; Chen, W.-Y.; Cheng, H.-Z.; Fu, S.-L. J. Phys. Chem. C 2010, 114, 15607-15611. doi:10.1021/jp1047985

29. Wang, K.; Hu, N.-X.; Xu, G.; Qi, Y. Carbon 2011, 49, 1769-1774. doi:10.1016/j.carbon.2010.12.063

30. Li, J.; Wang, L.; Jiang, W. Carbon 2010, 48, 2668-2671. doi:10.1016/j.carbon.2010.03.038

31. Wang, Z.; Koratkar, N.; Ci, L.; Ajayan, P. M. Appl. Phys. Lett. 2007, 90, 143117. doi:10.1063/1.2720761

32. Zhang, L.; Resasco, D. E. Langmuir 2009, 25, 4792-4798. doi:10.1021/la8040264

33. Bu, I. Y. Y.; Oei, S. P. Appl. Surf. Sci. 2010, 256, 6699-6704. doi:10.1016/j.apsusc.2010.04.073

34. Kakade, B.; Mehta, R.; Durge, A.; Kulkarni, S.; Pillai, V. Nano Lett. 2008, 8, 2693-2696. doi:10.1021/nl801012r

35. Li, S.; Li, H.; Wang, X.; Song, Y.; Liu, Y.; Jiang, L.; Zhu, D. J. Phys. Chem. B 2002, 106, 9274-9276. doi:10.1021/jp0209401

36. Li, Q.; DePaula, R.; Zhang, X.; Zheng, L.; Arendt, P. N.; Mueller, F. M.; Zhu, Y. T.; Tu, Y. Nanotechnology 2006, 17, 4533-4536. doi:10.1088/0957-4484/17/18/001

37. Chakrapani, N.; Wei, B.; Carrillo, A.; Ajayan, P. M.; Kane, R. S. Proc. Natl. Acad. Sci. U. S. A. 2004, 101, 4009-4012. doi:10.1073/pnas.0400734101

38. Bohn, S.; Platkiewicz, J.; Andreotti, B.; Adda-Bedia, M.; Couder, Y. Phys. Rev. E 2005, 71, 046215. doi:10.1103/PhysRevE.71.046215

39. Fusi, M.; Di Fonzo, F.; Casari, C. S.; Maccallini, E.; Caruso, T.; Agostino, R. G.; Bottani, C. E.; Bassi, A. L. Langmuir 2011, 27, 1935-1941. doi:10.1021/la103955q

40. Sauvage, F.; Di Fonzo, F.; Li Bassi, A.; Casari, C. S.; Russo, V.; Divitini, G.; Ducati, C.; Bottani, C. E.; Comte, P.; Graetzel, M. Nano Lett. 2010, 10, 2562-2567. doi:10.1021/nl101198b

41. De Nicola, F.; Castrucci, P.; Scarselli, M.; Nanni, F.; Cacciotti, I.; De Crescenzi, M. Sci. Rep. 2014, in press.

42. Clark, M. D.; Krishnamoorti, R. Thin Solid Films 2012, 520, 4332-4338. doi:10.1016/j.tsf.2012.02.067
43. Quéré, D. Nat. Mater. 2002, 1, 14-15. doi:10.1038/nmat715

44. De Gennes, P.-G.; Brochard-Wyart, F.; Quéré, D. Capillarity and wetting phenomena; Springer: New York, 2003.

45. Huang, L.; Lau, S. P.; Yang, H. Y.; Leong, E. S. P.; Yu, S. F. J. Phys. Chem. B 2005, 109, 7746-7748. doi:10.1021/jp046549s

46. Fürstner, R.; Barthlott, W.; Neinhuis, C.; Walzel, P. Langmuir 2005, 21 , 956-961. doi:10.1021/la0401011

47. Zhang, H.; Lamb, R.; Lewis, J. Sci. Technol. Adv. Mater. 2005, 6, 236-239. doi:10.1016/j.stam.2005.03.003

48. Lai, Y.; Tang, Y.; Gong, J.; Gong, D.; Chi, L.; Lin, C.; Chen, Z. J. Mater. Chem. 2012, 22, 7420-7426. doi:10.1039/c2jm16298a

49. Huang, Y.; Zhou, J.; Su, B.; Shi, L.; Wang, J.; Chen, S.; Wang, L.; Zi, J.; Song, Y.; Jiang, L. J. Am. Chem. Soc. 2012, 134, 17053-17058. doi:10.1021/ja304751k

50. Stalder, A. F.; Melchior, T.; Müller, M.; Sage, D.; Blu, T.; Unser, M. Colloids Surf., A 2010, 364, 72-81. doi:10.1016/j.colsurfa.2010.04.040

\section{License and Terms}

This is an Open Access article under the terms of the Creative Commons Attribution License

(http://creativecommons.org/licenses/by/2.0), which permits unrestricted use, distribution, and reproduction in any medium, provided the original work is properly cited.

The license is subject to the Beilstein Journal of

Nanotechnology terms and conditions:

(http://www.beilstein-journals.org/bjnano)

The definitive version of this article is the electronic one which can be found at: doi:10.3762/bjnano.6.34 